\title{
GMR
}

\section{Identification of altered pathways in hypertrophic cardiomyopathy based on combined data of protein-protein interactions and molecular pathways}

\author{
S.W. Zhang, J. Wang and J. Pan \\ Department of Cardiology, Linyi People's Hospital, Linyi, \\ Shandong Province, China \\ Corresponding author: J. Pan \\ E-mail: panjinlinyi@163.com \\ Genet. Mol. Res. 15 (2): gmr.15027754 \\ Received September 29, 2015 \\ Accepted December 22, 2015 \\ Published May 13, 2016 \\ DOI http://dx.doi.org/10.4238/gmr.15027754
}

\begin{abstract}
The purpose of our study was to identify molecular pathways altered during the pathogenesis of hypertrophic cardiomyopathy (HCM) based on data from the STRING proteinprotein interaction (PPI) database and the REACTOME pathway database. Identification of differentially expressed genes (DEGs) was carried out, followed by construction of a targeted network and selection of hub genes in this network. PPI pairs in each pathway were extracted, and altered pathways were identified when the said pathway differed from common interactions within the targeted network with a $\mathrm{P}$ value of less than 0.05 . These altered pathways were further validated based on enrichment of hub genes in pathways within the targeted network. Through this method, we identified 1085 DEGs. The DEGs were inputted into the STRING database, and the resulting targeted network was composed of 3631 interactions. Based on the selection criteria, 30 significantly changed pathways were screened in total. Among
\end{abstract}


these, the top five pathways were found to be involved in immune modulation, signal transduction, hemostasis, and G protein-coupled receptor signaling. Similarly, enrichment in hub gene interactions was also found in members within the altered pathways, including those involved in the innate immune system, the immune system, and signal transduction pathways. These altered pathways are important for understanding the underlying mechanisms of HCM, and can be used for clinical application of treatments in the future.

Key words: Hypertrophic cardiomyopathy; Targeted network; Molecular pathway; Protein-protein interaction

\section{INTRODUCTION}

Hypertrophic cardiomyopathy (HCM), characterized by heterogeneous phenotypes as well as genetic abnormalities, is a prevalent and familial cardiac disorder that can lead to sudden death (Maron, 2002; Seidman and Seidman, 2011). Approximately 1 of 4 patients with HCM demonstrates obstruction in the left ventricular outflow tract (Maron et al., 2003). It has been accepted that genetic variants and changes to certain molecular pathways may lead to HCM. To date, many genes and pathways resulting in sarcomeric mutations have been identified. These mutations may lead to myocyte hypertrophy and electrophysiological abnormalities that contribute to HCM. For example, more than $60 \%$ of genetic mutations in HCM patients occur in MYH7, MYL2, and MYBPC3, which have been shown to be sarcomeric genes (Efthimiadis et al., 2014; Roma-Rodrigues and Fernandes, 2014). Lin and Yun (2015) suggested that activation of the hypoxia-inducible factor pathway by hypoxia-inducible factor $2 \alpha$ is associated with HCM. Moreover, defects in the mitochondrial heme biosynthetic pathway have been shown to play important roles in early-onset fatal HCM (Antonicka et al., 2003). However, the potential mechanisms underlying the pathology of HCM are not fully understood. Efforts to elucidate the mechanisms of HCM have yielded paradoxical results. Thus, there is an urgent need to understand the molecular mechanism involved in HCM, and identification of altered pathways during disease onset may lead to greater understanding of HCM pathogenesis.

Pathway analysis is a bioinformatic tool used to screen abnormal pathways or modules in order to gain insights into the role of genes and proteins in disease pathogenesis (Glazko and Emmert-Streib, 2009). While this method can be used to reduce disease complexity, it is hard to fully interpret disease mechanisms using a single molecular method. Currently, network-based analysis has become more powerful in exploring disease mechanisms (Bradley et al., 2008). Recently, protein-protein interaction (PPI) networks, gene interaction networks, and transcriptome networks have proven to be effective in characterizing cellular processes in various diseases (Chen et al., 2013; Zhu et al., 2014; Zhang et al., 2015). PPI network analysis differs from pathway analysis in that it uses comprehensive networks to gain system-level biological meanings (Wu and Chen, 2009). To achieve biochemical functions, a combination of protein complexes and signaling pathways are needed (Wu et al., 2014). A pathway association network is a special form of "functional crosstalk networks" constructed from significant PPIs (Wu and Chen, 2012). Pathway association networks have been successfully used to study complex diseases, including cancer (Edelman et al., 2008) and Alzheimer's disease (Liu et al., 2010). These are various databases such as the search tool for the retrieval 
of interacting genes (STRING) (Franceschini et al., 2013) and REACTOME (Matthews et al., 2009), which provide data on PPIs and biological pathways.

Here, we present a novel approach to select altered pathways in HCM patients based on a combination of PPI and pathway information. The specific steps include identification of differentially expressed genes (DEGs), construction of targeted network, extraction of PPI pairs in each pathway, selection of altered pathways, and validation. The altered pathways were validated based on pathway enrichment of hub genes in the targeted network. A flowchart of this novel method used to predict altered biological pathways is presented in Figure 1.

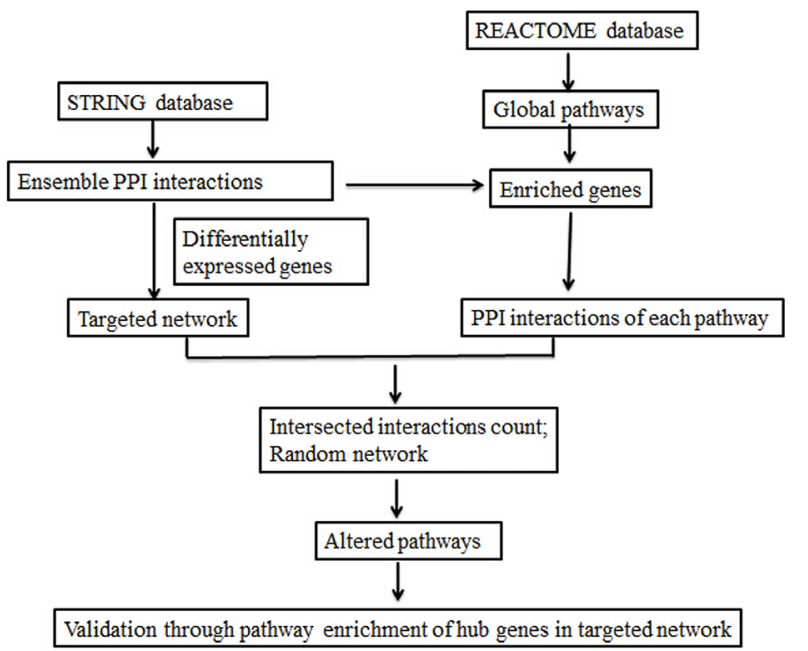

Figure 1. Flowchart for predicting altered pathways between hypertrophic cardiomyopathy patients and healthy controls.

\section{MATERIAL AND METHODS}

\section{Data collection}

The transcription profile of HCM patients and healthy controls (Accession No. E-GEOD-36961) was downloaded from the EMBL-EBI database (Hebl et al., 2012). In our study, gene microarray analysis was performed on heart tissues from $106 \mathrm{HCM}$ patients (54 males and 52 females, mean age at myectomy: $40.5 \pm 20.2$ years) and 39 healthy donors serving as controls. Assays were carried out using the A-GEOD-15389 platform of Illumina HumanHT-12 v3 Expression BeadChip.

\section{Data preprocessing and DEG selection}

The measured expression profiles were transformed into expression values using the robust multi-array average normalization approach (Parmigiani et al., 2003), and results were analyzed with $\mathrm{R}$ statistical package in the Bioconductor software. This processing included the following successive steps: background correction, quantile normalization of probe levels, and computation of expression measures by means of median polish analysis. 
For transcriptome profiling of HCM patients, the SAM package was utilized to identify DEGs. Briefly, the SAMR function of the SAM package was used to identify statistically significant gene expression. Each gene was given a score based on its change in expression when compared with its standard deviation. Genes with scores higher than the pre-determined limiting value were proposed to be potentially significant. The percentage of falsely positives relative to the significant genes was called the false discovery rate. To increase the stringency of our gene expression analysis, delta values were computed using SAMR. DEGs in HCM were screened according to the delta cut-off value of 5.37 .

\section{Construction of targeted network}

STRING is an online database and provides free access to experimental and predicted gene interactions (Szklarczyk et al., 2011). In the current study, the identified DEGs were inputted into the STRING database to obtain a network of corresponding proteins known as the "targeted network".

\section{Identification of PPIs in each pathway}

All PPIs in humans were downloaded from the STRING database. This ensemble of PPIs included 787,896 interactions. The REACTOME database includes extensive information on interconnecting pathways in Homo sapiens and many reference species, such as those generated by the genome projects (Croft, 2013). A total of 1675 human pathways were extracted from this database. Of note, pathways with gene set sizes of less than 1 were filtered out. Overall, 1639 pathways remained. Based on the genes contained in each pathway, human-specific PPIs in each pathway were extracted from ensemble PPIs.

\section{Identification of altered pathways}

In our study, we identified altered pathways based on the following two steps. First, similarity between interactions of targeted network and each pathway was measured. This was determined as the size of the common interaction labeled count $\left({ }_{i}\right)$, where count $\left({ }_{i}\right)$ was the number of common interactions between targeted network and each pathway, and $i$ was the $\mathrm{i}^{\text {th }}$ pathway. Second, random networks and $\mathrm{P}$ values were generated. Specifically, the DEGs identified (count $=\mathrm{A}$ ) were used to construct all possible PPI pairs $[\mathrm{B}=\mathrm{A} \times(\mathrm{A}-1) / 2]$. We generated 1000 random networks with the same size of interactions for the targeted network from B PPI pairs. Intersection of interactions between random network and each pathway were calculated. In light of these, the $\mathrm{P}$ value of each pathway was computed based on the following formula:

$$
P \text { value }=\operatorname{sum}\left[\operatorname{count}\left({ }_{\mathrm{ij}}\right)>\operatorname{count}\left(\left(_{\mathrm{i}}\right)\right] / 1000\right.
$$

where count $\left({ }_{\mathrm{ij}}\right)$ was the number of common interactions between random networks and each pathway, $\mathrm{i}$ represented the $\mathrm{i}^{\text {th }}$ pathway, and $\mathrm{j}$ denoted random times. The significantly changed pathways were screened with these thresholds: interactions $>15$ and a $\mathrm{P}$ value $<0.05$. 


\section{Identification of hub genes in targeted networks and selection of pathways related with hub genes}

The topological centralities, including the local scale (degree) and the global scale (between-ness, closeness, and stress), were extensively utilized in network analysis. Of these, degree is a simple and evident measure, which was identified as the number of links between a node with its neighboring nodes (Otte and Rousseau, 2002). In the present study, we studied the degree distribution of genes. Nodes with $\geq 39$ degrees were proposed to be hub genes.

Sub-networks composed of hub genes and their corresponding interactions were extracted from the targeted network. The intersection of interactions between the sub-network and each pathway was calculated and defined as count $\left({ }_{i}\right)$, where count $\left({ }_{i}\right)$ was the number of common interactions between the sub-network and the $\mathrm{i}^{\text {th }}$ pathway.

\section{RESULTS}

\section{Identification of DEGs and construction of targeted network}

We identified 1085 DEGs that were down-regulated, based on the delta cut-off value of 5.37. These 1085 DEGs were inputted into the STRING database. The targeted network was visualized, and involved 3631 interactions. In order to clearly present the targeted network, we selected interactions with combined scores of not less than 0.7 to construct the network. As shown in Figure 2, a total of 1399 interactions and 547 nodes were chosen to construct the network.

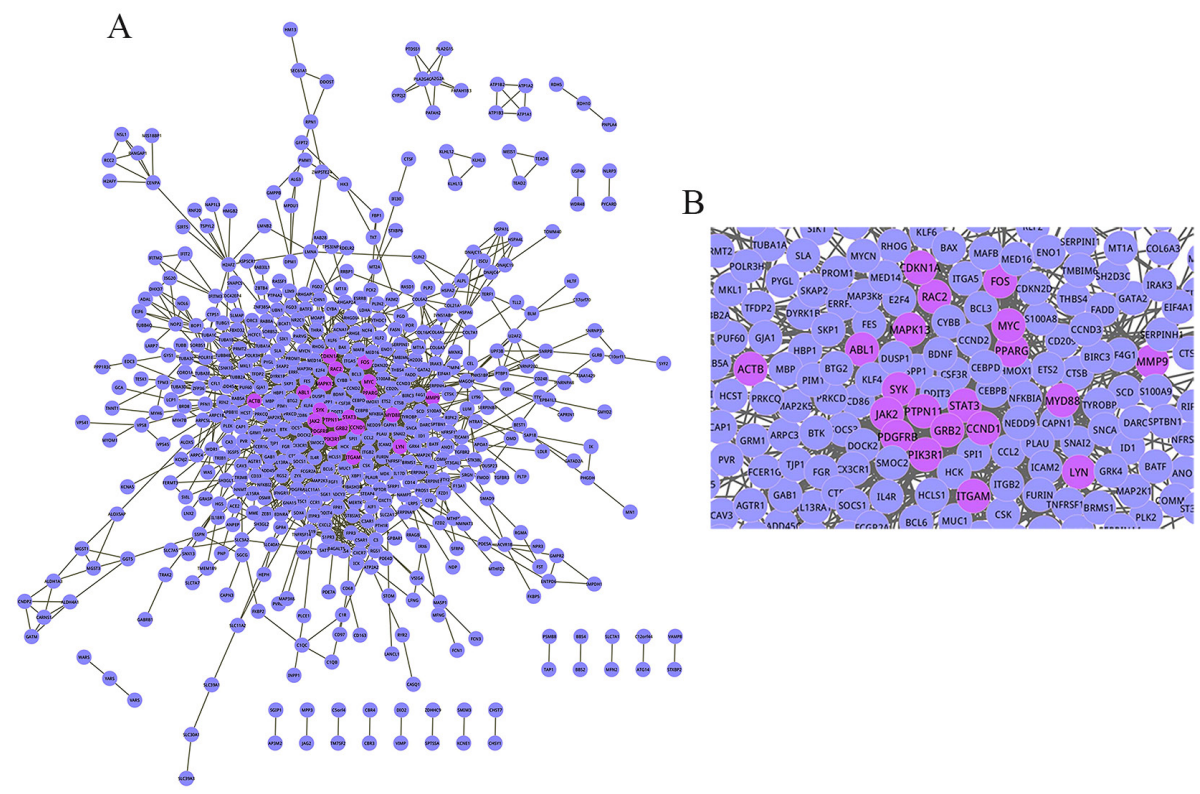

Figure 2. Targeted network composed of differentially expressed genes with combined edge scores of no less than 0.7. A. Whole targeted network, including 547 nodes and 1399 interactions. B. Magnified image of the target network. The nodes symbolize genes, pink nodes represent hub genes, and gene-gene interactions are represented by interconnecting lines. 


\section{Identification of altered pathways}

In our study, 1085 DEGs were used to construct all possible PPI pairs $(588,070)$. We generated 1000 random networks with 3631 interactions from the 588,070 PPI pairs. The intersections of interactions between random network and each pathway were obtained. The $P$ value of each pathway was computed and ranked in descending order. We identified 310 pathways with $\mathrm{P}$ value $<0.05$. Intriguingly, most of the $\mathrm{P}$ values approximated to zero or were equal to zero. Due to the similarity in $P$ values of these 310 pathways, interaction count may be another parameter that can be used to estimate the significance of pathways. Based on the common interactions between targeted network and our selection criteria, 30 significantly changed pathways were screened, as shown in Table 1. Among these, the top five significant pathways were found to be involved in the innate immune system, the overall immune system, signal transduction, hemostasis, and GPCR signaling.

Table 1. Disrupted pathways based on intersection $>15$ and $\mathrm{P}<0.05$.

\begin{tabular}{|c|c|}
\hline Pathway & Counts of common interactions between targeted network and each pathway \\
\hline Innate immune system & 80 \\
\hline Immune system & 76 \\
\hline Signal transduction & 48 \\
\hline Hemostasis & 47 \\
\hline Signaling by GPCR & 40 \\
\hline Disease & 38 \\
\hline GPCR downstream signaling & 36 \\
\hline Adaptive immune system & 33 \\
\hline Signaling by NGF & 31 \\
\hline Cell cycle & 29 \\
\hline NGF signaling via TRKA from the plasma membrane & 24 \\
\hline Fc epsilon receptor signaling & 21 \\
\hline Developmental biology & 20 \\
\hline Mitotic G1-G1/S phases & 20 \\
\hline Signaling by Rho GTPases & 20 \\
\hline Signaling by SCF-KIT & 20 \\
\hline Signaling by PDGF & 19 \\
\hline Activated TLR4 signaling & 18 \\
\hline Diseases of signal transduction & 18 \\
\hline Signaling by EGFR & 18 \\
\hline Toll like receptor 4 cascade & 18 \\
\hline Toll-like receptors cascades & 18 \\
\hline Transmembrane transport of small molecules & 18 \\
\hline Downstream signal transduction & 17 \\
\hline Metabolism & 17 \\
\hline Axon guidance & 16 \\
\hline DAP12 interactions & 16 \\
\hline DAP12 signaling & 16 \\
\hline Metabolism of proteins & 16 \\
\hline Signaling by ERBB4 & 16 \\
\hline
\end{tabular}

\section{Selection of hub genes in targeted network and pathways related with hub genes}

Among the 547 nodes in the targeted network, a total of 20 nodes with $>39$ degrees were identified, as depicted in Table 2. These included MYC (degree $=96), F O S($ degree $=88)$, 
STAT3 $($ degree $=83), C C N D 1($ degree $=73)$, and $A C T B($ degree $=71)$.

Sub-networks composed of hub genes and their corresponding interactions were extracted from the targeted network. Based on the intersection of interactions between subnetwork and each pathway, a total of 19 pathways were identified, as exhibited in Table 3. We found that these genes were involved in the immune system, innate immune system, NGF signaling, disease, and signal transduction. Based on these results, the innate immune system, immune system, and signal transduction pathways may play important roles in the pathogenesis of HCM.

Table 2. Hub genes in the targeted network with more than 40 degrees.

\begin{tabular}{|c|c|c|}
\hline Gene ID & Gene symbol & Degree \\
\hline 90 & $M Y C$ & 96 \\
\hline 82 & FOS & 88 \\
\hline 92 & STAT3 & 83 \\
\hline 125 & CCND1 & 73 \\
\hline 266 & $A C T B$ & 71 \\
\hline 136 & GRB2 & 63 \\
\hline 81 & JAK2 & 62 \\
\hline 135 & $A B L 1$ & 59 \\
\hline 93 & $R A C 2$ & 54 \\
\hline 122 & $M M P 9$ & 52 \\
\hline 167 & PTPN11 & 51 \\
\hline 169 & MAPK13 & 51 \\
\hline 310 & PIK3RI & 51 \\
\hline 104 & CDKNIA & 50 \\
\hline 119 & $P P A R G$ & 50 \\
\hline 166 & $L Y N$ & 47 \\
\hline 164 & PDGFRB & 45 \\
\hline 89 & ITGAM & 44 \\
\hline 694 & $S K Y$ & 44 \\
\hline 130 & MYD88 & 39 \\
\hline
\end{tabular}

Table 3. Disrupted pathways identified based on intersection $\geq 15$.

\begin{tabular}{l|l}
\hline Pathway & \multicolumn{1}{|c}{ Counts of common interactions between sub-network and each pathway } \\
\hline Immune system & 48 \\
\hline Innate immune system & 48 \\
\hline Signaling by NGF & 27 \\
\hline Disease & 26 \\
\hline Signal transduction & 26 \\
\hline NGF signaling via TRKA from the plasma membrane & 22 \\
\hline Hemostasis & 20 \\
\hline Adaptive immune system & 19 \\
\hline Signaling by SCF-KIT & 19 \\
\hline Fc epsilon receptor signaling & 18 \\
\hline Diseases of signal transduction & 17 \\
\hline Signaling by PDGF & 17 \\
\hline Cell cycle & 16 \\
\hline Downstream signal transduction & 16 \\
\hline Mitotic G1-G1/S phases & 16 \\
\hline DAP12 interactions & 15 \\
\hline DAP12 signaling & 15 \\
\hline Signaling by EGFR & 15 \\
\hline Signaling by ERBB4 & 15 \\
\hline
\end{tabular}




\section{DISCUSSION}

In order to gain greater insights into the molecular mechanisms of HCM, altered pathways were identified based on integrated data from PPI and human pathway information. Our results demonstrated that a total of 1085 DEGs were selected. Moreover, altered pathways were found to belong to the innate immune system, the overall immune system, and signal transduction pathways.

The immune system protects the host from invading pathogens and promotes tissue growth and repair during development and tissue injury. Regulatory $\mathrm{T}$ cells are found in the cardiac tissue and play important roles in modulating immune responses (Saxena et al., 2014). Kuusisto et al. (2012) demonstrated that $\mathrm{CD}^{+} \mathrm{T}$ cells were present in $\mathrm{HCM}$ patients, but were lacking in healthy subjects, suggesting that inflammation due to infiltrating immune cells is associated with HCM. Toll-like receptor 4 (TLR4) is a trans-membrane immune protein, and is present in almost all human immune and cardiac cells. It is associated with activation of inflammatory cytokines such as tumor necrosis factor- $\alpha$ (TNF- $\alpha$ ) and interleukin-6 (IL-6) (Kuusisto et al., 2012). Importantly, TNF- $\alpha$ and IL-6 can lead to the development of myocyte hypertrophy and fibrosis (Nian et al., 2004). As previously reported, myocardial fibrosis is a distinguishing feature of HCM (Elliott and McKenna, 2004). It has been proposed that activation of the innate immune system is a potential trigger for cardiomyopathy via cytokine activation (O'Neill and Bowie, 2007). In the present study, using a combination of PPI and pathway information, we were able to identify the specific pathways that differed between HCM patients and healthy controls. We found that pathways of the innate immune system were altered in HCM patients. This information can be used to expand our understanding on the pathophysiology of HCM. To the best of our knowledge, cardiac hypertrophy is a primary response to adapt to increased hemodynamic workload (Mondry and Swynghedauw, 1995). Mechanical stress is the main trigger that can induce the growth response in the overloaded myocardium. The MAPK signal transduction pathway is activated in response to most hypertrophy-associated stimuli (Molkentin and Dorn, 2001). Activation of MAPK signaling then induces downstream NF- $\mathrm{BB}$ signaling, which leads to recruitment of proinflammatory cell and release of pro-inflammatory cytokines in the myocardium (Nian et al., 2004; Leychenko et al., 2011). This can result in myocardial fibrosis. Similarly, NF-кB activation also mediates VEGF secretion in response to mechanical stretch stresses in the heart (Leychenko et al., 2011). VEGF is a growth factor that has been associated with hypertrophy of cardiomyocytes (Seko et al., 1999; Ruwhof and van der Laarse, 2000). The JAK/STAT pathway is a cytokine-activated signal transduction pathway that mediates the transduction of stress signals from the plasma membrane to the nucleus. In the heart, these stress signals are transduced by IL-6-type cytokines such as IL-6, IL-11, and leukemia inhibitory factor via glycoprotein 130, which predominantly activates STAT3 (Fischer and Hilfiker-Kleiner, 2007). STAT proteins, part of the JAK-STAT pathway, mediates the expression of genes encoding proteins involved in inflammation and extracellular matrix composition (Hilfiker-Kleiner et al., 2004). Of note, extracellular matrix composition accumulation is the basis of cellular fibrosis, which is a common characteristic in a variety of heart disorders including HCM and dilated cardiomyopathy (Khan and Sheppard, 2006). In light of these results, it is expected that signal transduction pathways are closely associated with HCM. Therefore, therapies directed at these altered signaling pathways may potentially alter the progression of HCM.

Although we were able to isolate several significant pathways in HCM, our study

Genetics and Molecular Research 15 (2): gmr.15027754

CFUNPEC-RP www.funpecrp.com.br 
was subjected to several limitations. The current study only employed bioinformatic tools to identify altered pathways during HCM pathogenesis, and our results require verification. Therefore, further experiments need to be carried out in order to validate our results and further explore the molecular mechanisms of HCM.

In conclusion, we identified altered biological pathways in the innate immune system, immune system, and signal transduction process in HCM patients. These results are important for understanding the underlying mechanisms of HCM and for clinical application of therapeutic strategies in the future.

\section{Conflicts of interest}

The authors declare no conflict of interest.

\section{ACKNOWLEDGMENTS}

We would like to thank the editor and reviewers for the critical comments and suggestions. We would like to thank Beijing Springer Medical Research Institute for the professional translation and paper polishing.

\section{REFERENCES}

Antonicka H, Mattman A, Carlson CG, Glerum DM, et al. (2003). Mutations in COX15 produce a defect in the mitochondrial heme biosynthetic pathway, causing early-onset fatal hypertrophic cardiomyopathy. Am. J. Hum. Genet. 72: 101-114.http://dx.doi.org/10.1086/345489

Bradley EW, Ruan MM, Vrable A and Oursler MJ (2008). Pathway crosstalk between Ras/Raf and PI3K in promotion of M-CSF-induced MEK/ERK-mediated osteoclast survival. J. Cell. Biochem. 104: 1439-1451. http://dx.doi. org $/ 10.1002 / \mathrm{jcb} .21719$

Chen J, Wang HY and Zeng CY (2013). Transcriptome network analysis of potential candidate genes for heart failure. Genet. Mol. Res. 12: 4687-4697.http://dx.doi.org/10.4238/2013.October.18.7

Croft D (2013). Building models using Reactome pathways as templates. Methods Mol. Biol. 1021: 273-283.http://dx.doi. org/10.1007/978-1-62703-450-0_14

Edelman EJ, Guinney J, Chi JT, Febbo PG, et al. (2008). Modeling cancer progression via pathway dependencies. PLOS Comput. Biol. 4: e28. http://dx.doi.org/10.1371/journal.pcbi.0040028

Elliott P and McKenna WJ (2004). Hypertrophic cardiomyopathy. Lancet 363: 1881-1891. http://dx.doi.org/10.1016/ $\underline{\text { S0140-6736(04)16358-7 }}$

Efthimiadis GK, Pagourelias ED, Gossios T and Zegkos T (2014). Hypertrophic cardiomyopathy in 2013: Current speculations and future perspectives. World J. Cardiol. 6: 26-37.http://dx.doi.org/10.4330/wjc.v6.i2.26

Fischer P and Hilfiker-Kleiner D (2007). Survival pathways in hypertrophy and heart failure: the gp130-STAT3 axis. Basic Res. Cardiol. 102: 279-297.http://dx.doi.org/10.1007/s00395-007-0658-z

Franceschini A, Szklarczyk D, Frankild S, Kuhn M, et al. (2013). STRING v9.1: protein-protein interaction networks, with increased coverage and integration. Nucleic Acids Res. 41: D808-D815.http://dx.doi.org/10.1093/nar/gks1094

Glazko GV and Emmert-Streib F (2009). Unite and conquer: univariate and multivariate approaches for finding differentially expressed gene sets. Bioinformatics 25: 2348-2354. http://dx.doi.org/10.1093/bioinformatics/btp406

Hebl VB, Bos JM, Oberg AL, Sun ZF, et al. (2012). Abstract 11099: transcriptome profiling of surgical myectomy tissue from patients with hypertrophic cardiomyopathy reveals marked overexpression of ACE2. Circulation 126: A11099.

Hilfiker-Kleiner D, Hilfiker A, Fuchs M, Kaminski K, et al. (2004). Signal transducer and activator of transcription 3 is required for myocardial capillary growth, control of interstitial matrix deposition, and heart protection from ischemic injury. Circ. Res. 95: 187-195. http://dx.doi.org/10.1161/01.RES.0000134921.50377.61

Khan R and Sheppard R (2006). Fibrosis in heart disease: understanding the role of transforming growth factor- $\beta$ in cardiomyopathy, valvular disease and arrhythmia. Immunology 118: 10-24. http://dx.doi.org/10.1111/j.13652567.2006.02336.x 
Kuusisto J, Kärjä V, Sipola P, Kholová I, et al. (2012). Low-grade inflammation and the phenotypic expression of myocardial fibrosis in hypertrophic cardiomyopathy. Heart 98: 1007-1013. http://dx.doi.org/10.1136/heartjnl-2011-300960

Leychenko A, Konorev E, Jijiwa M and Matter ML (2011). Stretch-induced hypertrophy activates NFkB-mediated VEGF secretion in adult cardiomyocytes. PLoS One 6: e29055.http://dx.doi.org/10.1371/journal.pone.0029055

Lin Q and Yun Z (2015). The hypoxia-inducible factor pathway in adipocytes: the role of HIF-2 in adipose inflammation and hypertrophic cardiomyopathy. Front. Endocrinol. (Lausanne) 6: 39.http://dx.doi.org/10.3389/fendo.2015.00039

Liu ZP, Wang Y, Zhang XS and Chen L (2010). Identifying dysfunctional crosstalk of pathways in various regions of Alzheimer's disease brains. BMC Syst. Biol. 4 (Suppl 2): S11.http://dx.doi.org/10.1186/1752-0509-4-S2-S11

Maron BJ (2002). Hypertrophic cardiomyopathy: a systematic review. JAMA 287: 1308-1320. http://dx.doi.org/10.1001/ jama.287.10.1308

Maron MS, Olivotto I, Betocchi S, Casey SA, et al. (2003). Effect of left ventricular outflow tract obstruction on clinical outcome in hypertrophic cardiomyopathy. N. Engl. J. Med. 348: 295-303.http://dx.doi.org/10.1056/NEJMoa021332

Matthews L, Gopinath G, Gillespie M, Caudy M, et al. (2009). Reactome knowledgebase of human biological pathways and processes. Nucleic Acids Res. 37: D619-D622.http://dx.doi.org/10.1093/nar/gkn863

Molkentin JD and Dorn GW, 2nd (2001). Cytoplasmic signaling pathways that regulate cardiac hypertrophy. Annu. Rev. Physiol. 63: 391-426.http://dx.doi.org/10.1146/annurev.physiol.63.1.391

Mondry A and Swynghedauw B (1995). Biological adaptation of the myocardium to chrome mechanical overload. Eur. Heart J. Suppl. 16: 64-73. http://dx.doi.org/10.1093/eurheartj/16.suppl_I.64

Nian M, Lee P, Khaper N and Liu P (2004). Inflammatory cytokines and postmyocardial infarction remodeling. Circ. Res. 94: 1543-1553. http://dx.doi.org/10.1161/01.RES.0000130526.20854.fa

O'Neill LA and Bowie AG (2007). The family of five: TIR-domain-containing adaptors in Toll-like receptor signalling. Nat. Rev. Immunol. 7: 353-364. http://dx.doi.org/10.1038/nri2079

Otte E and Rousseau R (2002). Social network analysis: a powerful strategy, also for the information sciences. J. Inf. Sci. 28: 441-453. http://dx.doi.org/10.1177/016555150202800601

Parmigiani G, Garrett ES, Irizarry RA and Zeger SL (2003). The analysis of gene expression data: methods and software. In: The analysis of gene expression data (Parmigiani G, Garrett ES, Irizarry RA and Zeger SL, eds.). Springer, New York, 1-45.

Roma-Rodrigues C and Fernandes AR (2014). Genetics of hypertrophic cardiomyopathy: advances and pitfalls in molecular diagnosis and therapy. Appl. Clin. Genet. 7: 195-208.

Ruwhof C and van der Laarse A (2000). Mechanical stress-induced cardiac hypertrophy: mechanisms and signal transduction pathways. Cardiovasc. Res. 47: 23-37.http://dx.doi.org/10.1016/S0008-6363(00)00076-6

Saxena A, Dobaczewski M, Rai V, Haque Z, et al. (2014). Regulatory T cells are recruited in the infarcted mouse myocardium and may modulate fibroblast phenotype and function. Am. J. Physiol. Heart Circ. Physiol. 307: H1233-H1242.http://dx.doi.org/10.1152/ajpheart.00328.2014

Seidman CE and Seidman JG (2011). Identifying sarcomere gene mutations in hypertrophic cardiomyopathy: a personal history. Circ. Res. 108: 743-750.http://dx.doi.org/10.1161/CIRCRESAHA.110.223834

Seko Y, Seko Y, Takahashi N, Shibuya M, et al. (1999). Pulsatile stretch stimulates vascular endothelial growth factor (VEGF) secretion by cultured rat cardiac myocytes. Biochem. Biophys. Res. Commun. 254: 462-465. http://dx.doi. org/10.1006/bbrc. 1998.9969

Szklarczyk D, Franceschini A, Kuhn M, Simonovic M, et al. (2011). The STRING database in 2011: functional interaction networks of proteins, globally integrated and scored. Nucleic Acids Res. 39: D561-D568. http://dx.doi.org/10.1093/ nar/gkq973

Wu X and Chen JY (2009). Molecular interaction networks: topological and functional characterizations. In: Automation in proteomics and genomics: An engineering case-based approach (Alterovitz G, Benson R and Ramoni M, eds.). John Wiley \& Sons, Ltd., Chichester, 145-174.

Wu X and Chen JY (2012). An evaluation for merging signaling pathways by using protein-protein interaction data. Genomic Signal Processing and Statistics, (GENSIPS), 2012 IEEE International Workshop on. IEEE. Washington, 203-206.

Wu X, Hasan MA and Chen JY (2014). Pathway and network analysis in proteomics. J. Theor. Biol. 362: 44-52. http:// dx.doi.org/10.1016/j.jtbi.2014.05.031

Zhang AM, Song H, Shen YH and Liu Y (2015). Construction of a gene-gene interaction network with a combined score across multiple approaches. Genet. Mol. Res. 14: 7018-7030. http://dx.doi.org/10.4238/2015.June.26.11

Zhu YC, Deng BY, Zhang LG, Xu P, et al. (2014). Protein-protein interaction network analysis of osteoarthritis-related differentially expressed genes. Genet. Mol. Res. 13: 9343-9351.http://dx.doi.org/10.4238/2014.November.11.1 